\title{
ON A TRAPEZOIDAL TYPE RULE FOR WEIGHTED INTEGRALS
}

\author{
ZHENG LIU
}

\begin{abstract}
An error runs through a paper by Cerone and Dragomir [1] is corrected. Thus enable us to get a right form of a trapezoidal type rule for weighted integrals and its applications in numerical integration.
\end{abstract}

\section{Preliminaries}

Some definitions are required to simplify the subsequent work.

Definition 1. Let $\omega(x)$ be a positive integrable function on $[a, b]$. Let $\mu$ and $\nu$ be its zeroth and first moments about zero so that

$$
\mu=\int_{a}^{b} \omega(x) d x<\infty
$$

and

$$
\nu=\int_{a}^{b} x \omega(x) d x<\infty
$$

Definition 2. $P$ and $Q$ will be used to denote the zeroth and first moments of $\omega(x)$ over a subinterval $[a, b]$. In particular, for $\lambda>0$ the subscript $a$ or $b$ will be used to indicate the intervals $[a, a+\lambda]$ and $[b-\lambda, b]$ respectively. Thus, for example,

and

$$
P_{a}=\int_{a}^{a+\lambda} \omega(x) d x
$$

$$
Q_{b}=\int_{b-\lambda}^{b} x \omega(x) d x .
$$

The following theorem is due to Hayashi [2, pp.331-312].

Theorem 1. Let $h:[a, b] \rightarrow \mathbb{R}$ be a nonincreasing mapping on $[a, b]$ and $g:[a, b] \rightarrow \mathbb{R}$ an integrable mapping on $[a, b]$ with

$$
0 \leq g(x) \leq A, \quad \text { for all } x \in[a, b] .
$$

Received January 6, 2003; revised March 12, 2003.

2000 Mathematics Subject Classification. 26D15, 41A55.

Key words and phrases. Hayashi inequality, Iyengar inequality, weighted quadrature rule. 
Then

$$
A \int_{b-\lambda}^{b} h(x) d x \leq \int_{a}^{b} h(x) g(x) d x \leq A \int_{a}^{a+\lambda} h(x) d x
$$

where

$$
\lambda=\frac{1}{A} \int_{a}^{b} g(x) d x
$$

Hayashi's inequality (1.3) will now be used to obtain inequalities for weighted integrals to give trapezoidal type quadrature rules.

\section{Trapezoidal Inequality for Weighted Integrals}

Lemma 1. Let $f: I \subseteq \mathbb{R} \rightarrow \mathbb{R}$ be a differentiable mapping on $\stackrel{\circ}{I}$ (the interior of $I$ ) and $[a, b] \subset \stackrel{\circ}{I}$ with $M=\sup _{x \in[a, b]} f^{\prime}(x)<\infty, m=\inf _{x \in[a, b]} f^{\prime}(x)>-\infty$ and $M>m$. Let $\omega(x) \geq 0$ for all $x \in[a, b]$ and $\mu=\int_{a}^{b} \omega(x) d x<\infty, \nu=\int_{a}^{b} x \omega(x) d x<\infty$ be the zeroth and first moments of $\omega(\cdot)$ on $[a, b]$. If $f^{\prime}$ is integrable on $[a, b]$ then the following inequality holds:

$$
\begin{aligned}
(M-m)\left[Q_{b}-(b-\lambda) P_{b}\right] & \leq \int_{a}^{b} \omega(x) f(x) d x-\mu(f(a)-m a)-m \nu \\
& \leq(M-m)\left[Q_{a}-(\lambda+a) P_{a}+\lambda \mu\right]
\end{aligned}
$$

where $P, Q$ are as describe in Definition 2 and $\lambda=\frac{b-a}{M-m}(S-m), S=\frac{f(b)-f(a)}{b-a}$.

Proof. Let $h_{b}(x)=\int_{x}^{b} \omega(u) d u$ and $g(x)=f^{\prime}(x)-m$. Then from Hayashi's inequality

$$
L_{b} \leq I_{b} \leq U_{b}
$$

where

$$
\begin{aligned}
I_{b} & =\int_{a}^{b} h_{b}(x)\left(f^{\prime}(x)-m\right) d x, \\
\lambda & =\frac{1}{M-m} \int_{a}^{b}\left(f^{\prime}(x)-m\right) d x,
\end{aligned}
$$

and

$$
\begin{aligned}
L_{b} & =(M-m) \int_{b-\lambda}^{b} h_{b}(x) d x, \\
U_{b} & =(M-m) \int_{a}^{a+\lambda} h_{b}(x) d x .
\end{aligned}
$$

Now, an integration by parts gives

$$
I_{b}=-\mu(f(a)-m a)-m \nu+\int_{a}^{b} \omega(x) f(x) d x .
$$


Also,

$$
\lambda=\frac{b-a}{M-m}(S-m)
$$

where

$$
S=\frac{f(b)-f(a)}{b-a}
$$

the slope of the secant over $[a, b]$.

It should be noted that $0<\lambda \leq b-a$ since $S \leq M$.

For the lower bound $L_{b}$ a change of order of integration gives

$$
\begin{aligned}
\frac{L_{b}}{M-m} & =\int_{b-\lambda}^{b} \omega(u) \int_{b-\lambda}^{u} d x d u \\
& =(\lambda-b) P_{b}+Q_{b}
\end{aligned}
$$

where $P_{b}$ and $Q_{b}$ are as describe in Definition 2.

Similarly, the upper bound $U_{b}$ may be obtained through a change of order of integration to give

$$
\begin{aligned}
\frac{U_{b}}{M-m} & =\int_{a}^{a+\lambda} \omega(u) \int_{a}^{u} d x d u+\int_{a+\lambda}^{b} \omega(u) \int_{a}^{a+\lambda} d x d u \\
& =\int_{a}^{a+\lambda}(u-a) \omega(u) d u+\lambda \int_{a+\lambda}^{b} \omega(u) d u \\
& =Q_{a}-(\lambda+a) P_{a}+\lambda \mu
\end{aligned}
$$

where $P_{a}$ and $Q_{a}$ are as describe in Definition 2 and $\mu$ is the zeroth moment of $\omega(x)$ on $[a, b]$.

Using (2.2)-(2.6) the lemma is thus proved.

Lemma 2. Let the conditions be as in Lemma 1 then the following inequality holds:

$$
\begin{aligned}
(M-m)\left[Q_{b}-(\lambda-b) P_{b}-\lambda \mu\right] & \leq \int_{a}^{b} \omega(x) f(x) d x-\mu(f(b)-m b)-m \nu \\
& \leq(M-m)\left[Q_{a}-(\lambda+a) P_{a}\right] .
\end{aligned}
$$

Proof. The proof follows along similar lines to that of Lemma 1.

Let $h_{a}(x)=-\int_{a}^{x} \omega(u) d u$ and $g(x)=f^{\prime}(x)-m$. Then using Hayashi's inequality (1.2) gives:

$$
L_{a} \leq I_{a} \leq U_{a}
$$

where

$$
I_{a}=\int_{a}^{b} h_{a}(x)\left(f^{\prime}(x)-m\right) d x
$$


and

$$
\begin{aligned}
L_{a} & =(M-m) \int_{b-\lambda}^{b} h_{a}(x) d x, \\
U_{a} & =(M-m) \int_{a}^{a+\lambda} h_{a}(x) d x .
\end{aligned}
$$

Now, a straight forward integration by parts yields

$$
I_{a}=-\mu(f(b)-m b)-m \nu+\int_{a}^{b} \omega(x) f(x) d x .
$$

Further, an interchange of the order of integration and simplification of results yields

$$
\frac{L_{a}}{M-m}=Q_{b}+(\lambda-b) P_{b}-\lambda_{\mu}
$$

and

$$
\frac{U_{a}}{M-m}=Q_{a}-(\lambda+a) P_{a} .
$$

Hence, using (2.8)-(2.11) the lemma is proved.

Theorem 2. Let the conditions of Lemmas 1 and 2 be maintained. Then the following inequality holds:

$$
\begin{aligned}
(M-m)\left[Q_{b}-(b-\lambda) P_{b}-\frac{\lambda}{2} \mu\right] & \leq \int_{a}^{b} \omega(x) f(x) d x-\frac{\mu}{2}[f(a)+f(b)-m(a+b)]-m \nu \\
& \leq(M-m)\left[Q_{a}-(\lambda+a) P_{a}+\frac{\lambda}{2} \mu\right]
\end{aligned}
$$

where the P's and $Q$ 's are as defined in Definition 2.

Proof. Addition of (2.1) and (2.7) produces (2.12) upon division by 2 .

Corollary 1. Let the conditions be as in the previous Lemmas and Theorem 2. Then,

$$
\begin{aligned}
\left|\int_{a}^{b} \omega(x) f(x) d x-\frac{\mu}{2}[f(a)+f(b)-m(a+b)]-m \nu\right| & \leq \frac{\mu}{2}(b-a)(S-m) \\
& \leq \frac{M-m}{2} \mu(b-a)
\end{aligned}
$$

where $S$ is the slope of the secant on $[a, b]$.

Proof. The corollary follows readily from (2.12) on noting that

$$
\begin{aligned}
& Q_{b}=\int_{b-\lambda}^{b} x \omega(x) d x \geq(b-\lambda) \int_{b-\lambda}^{b} \omega(x) d x, \\
& Q_{a}=\int_{a}^{a+\lambda} x \omega(x) d x \leq(\lambda+a) \int_{a}^{a+\lambda} \omega(x) d x
\end{aligned}
$$


and substituting $(M-m) \lambda=(b-a)(S-m)$.

Remark 1. Allowing $\omega(x) \equiv 1$ in (2.12) gives from Definitions 1 and 2

$$
\mu=b-a, \quad \nu=\frac{b^{2}-a^{2}}{2}, \quad P_{a}=P_{b}=\lambda, \quad Q_{a}=\frac{\lambda}{2}(\lambda+2 a) \text { and } Q_{b}=\frac{\lambda}{2}(2 b-\lambda) .
$$

This reveals the lower bound to be negative the upper bound and we have the result of Cerone and Dragomir [3] as

$$
\begin{aligned}
\left|\int_{a}^{b} f(x)-\frac{b-a}{2}[f(a)+f(b)]\right| & \leq \frac{(b-a)^{2}}{2(M-m)}(S-m)(M-S) \\
& \leq \frac{M-m}{2}\left(\frac{b-a}{2}\right)^{2}
\end{aligned}
$$

where $S=\frac{f(b)-f(a)}{b-a}$. It should be mentioned that (2.14) is first proved by Agarwal and Dragomir [4] which is a generalization of the well known Iyengar inequality [5].

Remark 2. The bounds in (2.12) are not symmetric in general since for this to be so they must sum to zero. Let $L_{1}$ be the lower bound and $U_{1}$ be the upper bound. Then

$$
U_{1}+L_{1}=(M-m)\left[\left(Q_{b}-(b-\lambda) P_{b}\right)-\left((\lambda+a) P_{a}-Q_{b}\right)\right] .
$$

We know from the proof of Corollary 1 that $Q_{b} \geq(b-\lambda) P_{b}$ and $Q_{a} \leq(\lambda+a) P_{a}$, so $U_{1}+L_{1}=0$ when $Q_{b}-(b-\lambda) P_{b}=(\lambda+a) P_{a}-Q_{a}$.

Lemma 3. Let the Conditions of Theorem 2 and Lemmas 1 and 2 hold. Then, for $\omega(x)$ symmetric about the mid-point $\frac{a+b}{2}$, the bounds in (2.12) are symmetric. Hence

$$
\begin{aligned}
& \left|\int_{a}^{b} \omega(x) f(x) d x-\frac{\mu}{2}[f(a)+f(b)-m(a+b)]-m \nu\right| \\
\leq & (M-m)\left[\frac{\lambda}{2} \mu-\int_{0}^{\lambda} u \omega(\lambda+a-u) d u\right] .
\end{aligned}
$$

Proof. From Remark 2 and Definition 2, the sum of the upper and lower bounds in (2.12), $U_{1}$ and $L_{1}$ respectively is:

$$
\begin{aligned}
U_{1}+L_{1} & =(M-m)\left[\int_{b-\lambda}^{b}[x-(b-\lambda)] \omega(x) d x-\int_{a}^{a+\lambda}(\lambda+a-x) \omega(x) d x\right] \\
& =(M-m)\left[\int_{0}^{\lambda} u \omega(b-\lambda+u) d u-\int_{0}^{\lambda} u \omega(\lambda+a-u) d u\right] .
\end{aligned}
$$

Now,

$$
U_{1}+L_{1}=(M-m) \int_{0}^{\lambda} u\left[\omega\left(\frac{a+b}{2}+z\right)-\omega\left(\frac{a+b}{2}-z\right)\right] d u
$$


where $z=\frac{b-a}{2}-\lambda+u$.

Thus

$U_{1}+L_{1}=(M-m) \int_{\frac{b-a}{2}-\lambda}^{\frac{b-a}{2}}\left(z+\lambda-\frac{b-a}{2}\right)\left[\omega\left(\frac{a+b}{2}+z\right)-\omega\left(\frac{a+b}{2}-z\right)\right] d z=0$

for $\omega(\cdot)$ symmetric about $\frac{a+b}{2}$. Hence the bounds in (2.12) are symmetric.

Now, from the upper bound in (2.12), $U_{1}$ is such that

$$
\begin{aligned}
\frac{U_{1}}{M-m} & =\frac{\lambda}{2} \mu-\left[(\lambda+a) P_{a}-Q_{a}\right] \\
& =\frac{\lambda}{2} \mu-\int_{a}^{a+\lambda}(\lambda+a-x) \omega(x) d x \\
& =\frac{\lambda}{2} \mu-\int_{0}^{\lambda} u \omega(\lambda+a-u) d u .
\end{aligned}
$$

Thus, the lemma is proved.

It should be noted that the expression for $U_{1}$ obtained above may be written as

$$
\begin{aligned}
\frac{U_{1}}{M-m} & =\frac{\lambda}{2} \mu-\int_{0}^{\lambda} u \omega\left(\frac{a+b}{2}-z\right) d z \\
& =\frac{\lambda}{2} \mu-\int_{0}^{\lambda} u \omega\left(z-\frac{a+b}{2}\right) d z
\end{aligned}
$$

where $z=u+\frac{b-a}{2}-\lambda$. Here, we are using the fact that the weight function $\omega(\cdot)$ is symmetric about the mid-point.

Corollary 2. Let the conditions be as in the previous lemmas and Theorem 2. Then

$$
\begin{aligned}
& (M-m)\left[Q_{b}-(b-\lambda) P_{b}\right]-\mu\left[\left(\frac{b-a}{2}\right) S+a m\right]+m \nu \\
\leq & \int_{a}^{b} \omega(x) f(x) d x-\frac{\mu}{2}[f(a)+f(b)] \\
\leq & (M-m)\left[Q_{a}-(\lambda+a) P_{a}\right]+\mu\left[\left(\frac{b-a}{2}\right) S-b m\right]+m \nu .
\end{aligned}
$$

Proof. A simple rearrangement of the terms in (2.12), collecting the coefficient of $\mu$ and using the fact that $(M-m) \lambda=(b-a)(S-m)$ produces the result.

Remark 3. Using similar approximation as those in Corollary 1, simpler bounds may be obtained viz.,

$$
\begin{aligned}
& m \nu-\mu\left[\left(\frac{b-a}{2}\right) S+a m\right] \\
\leq & \int_{a}^{b} \omega(x) f(x) d x-\frac{\mu}{2}[f(a)+f(b)] \leq m \nu+\mu\left[\left(\frac{b-a}{2}\right) S-b m\right] .
\end{aligned}
$$




\section{Application in Numerical Integration}

In this section we will demonstrate how the results obtained in Section 2 may be utilized to obtain quadrature rules for weighted functions.

Theorem 3. Let $f:[a, b] \rightarrow \mathbb{R}$ be a differentiable mapping on $(a, b)$ with $M=$ $\sup _{x \in[a, b]} f^{\prime}(x)<\infty, m=\inf _{x \in[a, b]} f^{\prime}(x)>-\infty$, and $M>m$. Let $I_{n}$ be a partition of $[a, b]$ such that $I_{n}: a=x_{0}<x_{1}<\cdots<x_{n-1}<x_{n}=b$. Further, let $\omega(x) \geq 0$ for all $x \in[a, b]$ and $\mu=\int_{a}^{b} \omega(x) d x<\infty, \nu=\int_{a}^{b} x \omega(x) d x<\infty$ be the zeroth and first moments of $\omega(\cdot)$ on $[a, b]$. Then, the following weighted quadrature rule holds

$$
\int_{a}^{b} \omega(x) f(x) d x=A\left(\omega, f, I_{n}\right)+R\left(\omega, f, I_{n}\right)
$$

where $A\left(\omega, f, I_{n}\right)$ is an approximation to the weighted integral. Namely,

$$
\begin{aligned}
A\left(\omega, f, I_{n}\right) & =\frac{1}{2} \sum_{i=0}^{n-1} \mu_{i}\left[f\left(x_{i}\right)+f\left(x_{i+1}\right)-m\left(x_{i}+x_{i+1}\right)\right]+m \nu \\
& =\frac{1}{2}\left[\mu_{0} g_{0}+\mu_{n-1} g_{n}+\sum_{i=1}^{n-1}\left(u_{i-1}+\mu_{i}\right) g_{i}\right]+m \nu
\end{aligned}
$$

with $g_{i}=f\left(x_{i}\right)-m x_{i}, u_{i}=\int_{x_{i}}^{x_{i+1}} \omega(x) d x, \nu_{i}=\int_{x_{i}}^{x_{i+1}} x \omega(x) d x, i=0,1, \ldots, n-1$. In addition, the remainder term $R\left(\omega, f, I_{n}\right)$ satisfies

$$
\begin{aligned}
\left|R\left(\omega, f, I_{n}\right)\right| & \leq \frac{1}{2} \sum_{i=0}^{n-1} \mu_{i}\left[f\left(x_{i+1}\right)-f\left(x_{i}\right)-m\left(x_{i+1}-x_{i}\right)\right] \\
& =\frac{1}{2}\left[\mu_{n-1} g_{n}-\mu_{0} g_{0}+\sum_{i=1}^{n-1}\left(\mu_{i-1}-\mu_{i}\right) g_{i}\right] \\
& \leq \frac{M-m}{2} \sum_{i=0}^{n-1} \mu_{i} h_{i},
\end{aligned}
$$

where $h_{i}=x_{i+1}-x_{i}$.

Proof. Applying inequality (2.13) of Corollary 1 on the interval $\left[x_{i}, x_{i+1}\right]$ for $i=$ $0,1, \ldots, n-1$ we have

$$
\begin{aligned}
& \left|\int_{x_{i}}^{x_{i+1}} \omega(x) f(x) d x-\frac{\mu_{i}}{2}\left[f\left(x_{i}\right)+f\left(x_{i+1}\right)-m\left(x_{i}+x_{i+1}\right)\right]-m \nu_{i}\right| \\
\leq & \frac{\mu_{i}}{2}\left[f\left(x_{i+1}\right)-f\left(x_{i}\right)-m\left(x_{i+1}-x_{i}\right)\right] .
\end{aligned}
$$

Summing over $i$ for $i=0,1, \ldots, n-1$ gives the quadrature rule

$$
A\left(\omega, f, I_{n}\right)=\frac{1}{2} \sum_{i=0}^{n-1} \mu_{i}\left[f\left(x_{i}\right)+f\left(x_{i+1}\right)-m\left(x_{i}+x_{i+1}\right)\right]+m \sum_{i=0}^{n-1} \nu_{i}
$$




$$
=\frac{1}{2} \sum_{i=0}^{n-1} \mu_{i}\left(g_{i}+g_{i+1}\right)+m \nu
$$

where $g_{i}=f\left(x_{i}\right)-m x_{i}$.

Hence

$$
A\left(\omega, f, I_{n}\right)=\frac{1}{2}\left[\mu_{0} g_{0}+\mu_{n-1} g_{n}+\sum_{i=1}^{n-1}\left(\mu_{i-1}+\mu_{i}\right) g_{i}\right]+m \nu .
$$

The remainder term $R\left(\omega, f, I_{n}\right)$ is such that

$$
\begin{aligned}
\left|R\left(\omega, f, I_{n}\right)\right| & \leq \frac{1}{2} \sum_{i=0}^{n-1} \mu_{i}\left[f\left(x_{i+1}\right)-f\left(x_{i}\right)-m\left(x_{i+1}-x_{i}\right)\right] \\
& =\frac{1}{2} \sum_{i=0}^{n-1} \mu_{i}\left[g_{i+1}-g_{i}\right] \\
& =\frac{1}{2}\left[\mu_{n-1} g_{n}-\mu_{0} g_{0}+\sum_{i=1}^{n-1}\left(\mu_{i-1}-\mu_{i}\right) g_{i}\right] .
\end{aligned}
$$

Using the second inequality in Corollary 1 gives

$$
\left|R\left(\omega, f, I_{n}\right)\right| \leq \frac{M-m}{2} \sum_{i=0}^{n-1} \mu_{i} h_{i} .
$$

Hence the theorem is proved.

If a uniform grid is taken so that $x_{i}=x_{0}+i h, i=0,1, \ldots, n$, then

$$
\left|R\left(\omega, f, I_{n}\right)\right| \leq \frac{M-m}{2} h \mu .
$$

\section{References}

[1] P. Cerone and S. S. Dragomir, On a weighted generalization of Iyengar type inequalities involving bounded first derivative, Mathematical Inequalities \& Applications 3(2000), 3544 .

[2] D. S. Mitrinović, J. E. Pečarić and A. M. Fink, Classical and New Inequalities in Analysis, Kluwer Academic Publishers, 1993.

[3] P. Cerone and S. S. Dragomir, Lobatto type quadrature rules for functions with bounded derivative, RGMIA Research Report Collection 2(1999), 133-146.

[4] R. P. Agarwal and S. S. Dragomir, An application of Hayashi's inequality for differentiable functions, Computers Math. Appl. 32(1996), 95-99.

[5] K. S. K. Iyengar, Note on an inequality, Math. Student 6(1938), 75-76.

Institute of Applied Mathematics, Faculty of Science, Anshan University of Science and Technology, Anshan 114044, Liaoning, People's Republic of China. 